\title{
Construção e validação de uma ficha clínica para acompanhamento de pré- natal de risco habitual*
}

\author{
Construction and validation of a clinical form for usual-risk prenatal follow-up* \\ Construcción y validación de una ficha clínica para el monitoreo del riesgo prenatal habitual *
}

\section{Daniela Aparecida Almeida Duque ${ }^{\mathrm{I}}$, Betânia Maria Fernandes (in memoriam) ${ }^{\mathrm{II}}$}

Resumo: Objetivo: construir e validar uma ficha clínica para acompanhamento do pré-natal de risco habitual. Método: pesquisa metodológica com emprego de Técnica Delphi para validar o instrumento quanto a pertinência/representatividade, aplicando-se o cálculo do coeficiente de validade de conteúdo, cujo valor mínimo adotado foi $\geq 80 \%$. Os critérios de seleção dos participantes consistiram em ser enfermeiro, docente de instituição pública com título de Doutor e especialista em Obstetrícia. O primeiro painel foi constituído por 15 juízes e o segundo por 13. O estudo foi realizado entre fevereiro a junho de 2016. Resultados: o cálculo do Coeficiente de Validação de Conteúdo mensurou a Pertinência/Representatividade de cada item da ficha clínica mediante a análise de dois painéis, os quais alcançaram o coeficiente estabelecido. Conclusão: a ficha clínica validada está apta para aplicação em consultas de pré-natal de risco habitual.

Descritores: Cuidado pré-natal; Ficha clínica; Gravidez; Tecnologia; Assistência de Enfermagem

\begin{abstract}
Objective: to build and validate a clinical form record for usual-risk prenatal follow-up. Method: methodological research with employment of Delphi Technique to validate the instrument as the relevance/representativeness, by applying the calculation of the content validity coefficient, whose minimum value adopted was $\geq 80 \%$. The criteria for the selection of participants consisted of being a nurse, professor at government institution with the degree of $\mathrm{PhD}$ and specialist in Obstetrics. The first panel was composed of 15 judges and the second, of 13. The study was conducted between February and June 2016. Results: the calculation of the Content Validity Coefficient measured the Relevance/Representativeness of each item of the clinical form through the analysis of two panels, which reached the coefficient set. Conclusion: the validated clinical form is suitable for application in usual-risk prenatal consultations.
\end{abstract}

Descriptors: Prenatal care; Clinical form; Pregnancy; Technology; Nursing Care

Resumen: Objetivo: construir y validar una ficha clínica para el monitoreo del riesgo prenatal habitual. Método: investigación metodológica con el empleo de la Técnica Delphi para validar el instrumento de acuerdo con la

\footnotetext{
I Enfermeira. Mestre em Enfermagem pela Universidade Federal de Juiz de Fora. Juiz de Fora, Minas Gerais, Brasil. E-mail: danielaalmeidaduque@gmail.com ORCID: https://orcid.org/0000-0002-3657-2954

II Enfermeira. Mestre em Enfermagem pela Universidade Federal de Juiz de Fora. Juiz de Fora, Minas Gerais, Brasil. E-mail: danielaalmeidaduque@gmail.com ORCID: https://orcid.org/0000-0002-3657-2954
} 
pertinencia/representatividad, aplicando el cálculo del coeficiente de validación de contenido, cuyo valor mínimo aprobado era $\geq 80 \%$. Los criterios para la selección de los participantes consistían en ser un(a) enfermero(a), profesor(a) de institución pública con el título de Doctor(a) y especialista en Obstetricia. El primer panel estuvo integrado por 15 jueces y el segundo, por 13. El estudio se realizó entre febrero y junio de 2016. Resultados: el cálculo del Coeficiente de Validación de Contenido midió la pertinencia/representación de cada elemento de la forma clínica a través del análisis de dos paneles, que alcanzaron el coeficiente establecido. Conclusión: la ficha clínica validada es adecuada para su aplicación en las consultas prenatales de riesgo habitual.

Descriptores: Forma clínica; Atención prenatal; Embarazo; Tecnología; Cuidados de enfermería

\section{Introdução}

O pré-natal tem como propósito assistir à evolução da gestação, intervindo em situações de risco que podem acometer ao binômio mamãe-bebê, com um acompanhamento que engloba ações de promoção à saúde, prevenção e apoio psicossocial. O início precoce do pré-natal até a $12^{\underline{a}}$ semana de gestação constitui um indicador de qualidade da assistência. ${ }^{1}$

Os cuidados dedicados à fase gestacional visam reduzir a morbimortalidade materna e neonatal, uma vez que o índice de mortalidade é um importante indicador de saúde da mulher, remetendo às disparidades em uma região/país, principalmente em países em desenvolvimento, nos quais ocorrem $99 \%$ dos óbitos. Os índices elevados são um desafio para a Saúde Pública, pois um número significante de mortes é decorrente de causas obstétricas diretas passíveis de identificação e tratamento pela (o) enfermeira (o) durante a assistência pré-natal e puerperal em nível primário de atenção à saúde. ${ }^{2-3}$

Segundo a Organização Mundial de Saúde (OMS) o Brasil conseguiu reduzir 56\% da mortalidade de 1990 a 2015, de 140 por 100 mil nascidos vivos para 62 por 100 mil nascidos vivos. No mundo esta queda foi de $45 \%$, de 380 por 100 mil nascidos vivos para 210 por 100 mil nascidos vivos. O relatório da OMS revela que a mortalidade nos países em desenvolvimento é 14 vezes maior do que em países desenvolvidos. Espera-se que entre 2016 e 2030 a mortalidade mundial seja inferior a 70 óbitos por 100 mil nascidos vivos, compreendendo um dos objetivos do desenvolvimento sustentável passiveis de ser reduzido por meio do início precoce do prénatal e um acompanhamento integral até o puerpério..$^{2-3}$ 
O pré-natal consiste em um acompanhamento imprescindível para o direcionamento de condutas mediante uma avaliação clínica obtida nas consultas capaz de contribuir com a redução dos índices de mortalidade materna e perinatal. Entretanto, o país apresenta discrepâncias entre o que é preconizado nas políticas e sua execução real, pois se constata descumprimento no que tange o acesso ao pré-natal, número de consultas e realização completa das etapas do exame físico, obstétrico, disponibilidade de exames laboratoriais e de imagem, o que repercute na qualidade e resolutividade da assistência. ${ }^{4-5}$

Constituem obstáculos para a adesão ao pré-natal a situação financeira, baixa escolaridade, ser solteira e ser multíparas (eleva o risco em duas vezes). Além disso, o estado de saúde ou a distância da residência à Atenção Básica podem exigir gastos com transporte e dificultar a presença às consultas. ${ }^{6}$ A insatisfação com a equipe de saúde, a duração da consulta, a influência de fatores culturais, a ausência ou dificuldade na oferta de medicamentos e exames no serviço de saúde podem refletir nas perspectivas que a usuária tem com a assistência disponibilizada ao ciclo gravídico. ${ }^{7}$

Dessa forma, a consulta de pré-natal de risco habitual é definida como uma assistência à gestante sem comorbidades pregressas ou atuais que constituam risco de instabilidades capazes de resultar em desfechos indesejados ao binômio, o que exige acompanhamento médico. Entretanto, o pré-natal de gestantes sem complicações poderá ser realizado pela (o) enfermeira (o) e intercalado com o médico. Ressalta-se que no contexto da Atenção Primária à Saúde a enfermeira solicitará exames e prescreverá medicações conforme protocolo adotado pelo município, será responsável pela prescrição de vacinas, identificação de sinais de riscos e encaminhamentos pertinentes à atenção secundária e ou terciária. ${ }^{8}$

A consulta de enfermagem foi aprovada pelo Conselho Federal de Enfermagem (COFEN) por meio da resolução № 159/1993 por ser uma Ciência com domínio científico que confere autonomia aos profissionais para implementar as ações presentes na lei do exercício 
profissional e realizar a tomada de decisão frente aos eventos de saúde e doença. ${ }^{9}$ Trata-se de uma oportunidade para construir um histórico do usuário com elevado número de informações, dado que o vínculo estabelecido facilita o diálogo e direciona o plano terapêutico. ${ }^{10}$

$\mathrm{O}$ investimento em pesquisas na área da enfermagem que visam à introdução e desenvolvimento tecnológico constituem um desafio ao avanço nos modos de cuidar, constituindo uma das prioridades da Agenda Nacional de Prioridades de Pesquisa em Saúde com destaque para estudos regionais e nacionais. ${ }^{11} \mathrm{~A}$ adesão às fichas clínicas para registrar as condutas e informações adquiridas nas consultas é fundamental para sistematizar as etapas do pré-natal, incluindo a gestação, o parto, o recém-nascido e o puerpério. Todavia, os instrumentos precisam contemplar itens que assegurem um atendimento amplo e apto para alertar o profissional quanto à presença de risco, o que favorece as intervenções e pode contribuir com a redução da mortalidade materna e perinatal. ${ }^{12}$

Diante do exposto, surgiu o interesse em desenvolver esta pesquisa por considerar que o instrumento específico para a consulta de enfermagem no pré-natal de risco habitual poderá ser uma tecnologia capaz de contribuir com o cuidado da (o) enfermeira (o) na assistência à gestante na Atenção Primária à Saúde, de forma eficaz, fundamentado nas normas recomendadas pela Organização Pan Americana de Saúde e Ministério da Saúde para a consulta à gestante no prénatal de risco habitual. Ressalta-se que o emprego de tecnologias na área da saúde aumenta a qualidade do cuidado e facilita os processos desde as atividades técnicas que compreendem a assistência até a gestão dos espaços de saúde.

A atuação da enfermagem em todas as etapas encontra-se atrelada ao uso de tecnologias, que podem sem classificadas como leves, traduzidas como o ato de cuidar de pessoas e as relações interpessoais estabelecidas entre o cliente e o profissional; leve-dura referindo-se à criação de modelos de cuidados a partir de bases científicas; e dura sendo representada pelos equipamentos tecnológicos. ${ }^{13}$ 
Nesse contexto, a adaptação e validação de um instrumento para a consulta de enfermagem no pré-natal enquadra-se na classificação de tecnologia leve-dura, por ser capaz de favorecer uma investigação ampla que facilita o diálogo entre a (o) enfermeira (o) e a usuária, de modo a identificar fatores de riscos relevantes para o desenvolvimento gestacional. Além de possibilitar a realização de encaminhamentos em tempo hábil, educação em saúde, esclarecimento de dúvidas e a conquista da confiança da gestante.

Dessarte, o objeto desta investigação consiste na adaptação de uma ficha clínica para a consulta de enfermagem no pré-natal de risco habitual na Atenção Primária à Saúde. Objetivase construir e validar uma ficha clínica para acompanhamento do pré-natal de risco habitual.

\section{Método}

Pesquisa metodológica com emprego da Técnica Delphi para validar um instrumento para a consulta de enfermagem no pré-natal de risco habitual. O estudo metodológico oportuniza explorar métodos que visam selecionar e organizar dados por meio da elaboração de instrumentos que devem ser submetidos às etapas de validação. A técnica Delphi consiste em um método sistematizado para consolidar informações a partir do consenso de especialistas, os quais podem ser denominados juízes, peritos, especialistas, respondentes, painelistas ou experts. ${ }^{14}$

A construção da ficha clínica ocorreu após revisão minuciosa de literatura e observação dos instrumentos recomendados pelo Centro Latino-Americano de Perinatologia, Saúde da Mulher e Reprodutiva da Organização Pan Americana da Saúde/Organização Mundial da Saúde e Ministério da Saúde do Brasil. Assim, estabeleceram-se as informações relevantes e os conteúdos que passaram pelo processo de validação, ou seja, os participantes verificaram se o instrumento é capaz de proporcionar respostas confiáveis e precisas dentro da temática estudada. ${ }^{14-15}$ 
Os critérios de seleção para participar da pesquisa foi uma amostra não aleatória de enfermeiro (a) docente e especialista em enfermagem obstétrica, grau acadêmico de Doutor, atuando em universidades públicas do país, de forma que obtivessem representantes de todas as regiões, como requisito para validar o instrumento e garantir constructos compatíveis com as variâncias regionais. Foram considerados participantes as (os) enfermeiras (os) especialistas com no mínimo 02 anos de ensino na área de saúde da mulher, identificados a partir do currículo disponível na Plataforma Lattes e por meio da técnica de bola de neve (snowball). Essa técnica recruta novos participantes a partir da indicação de um anterior e assim sucessivamente, até que seja alcançado o objetivo proposto. ${ }^{16}$

As etapas que constituem a Técnica Delphi estão apresentadas na Figura 1:

Figura 1 - Técnica Delphi 


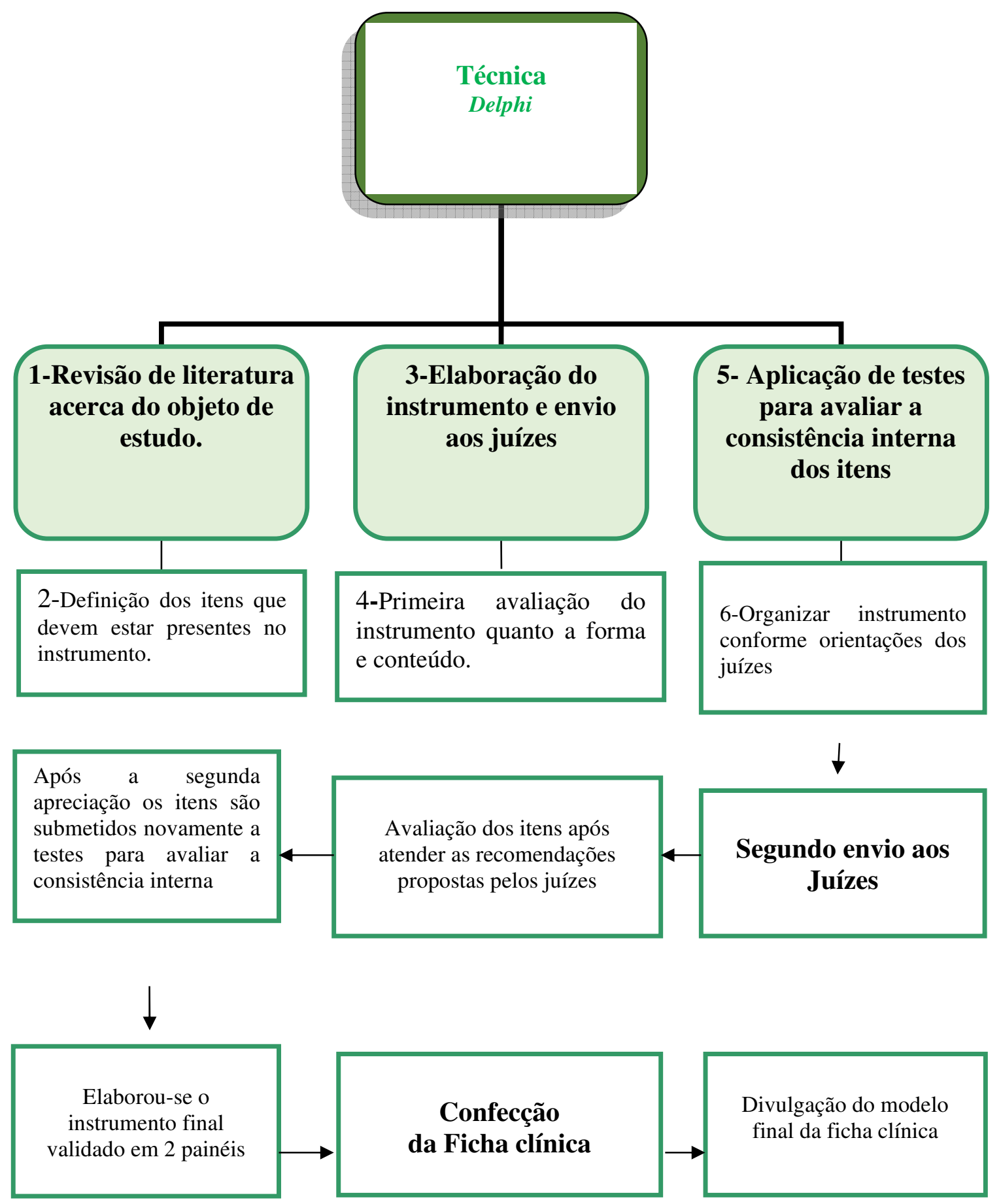

Fonte: Elaborado pelas autoras.

A etapa de validação do primeiro painel ocorreu entre 15 de fevereiro a 25 março de 2016. Após análise curricular foram considerados elegíveis 63 profissionais, porém 33 e-mails enviados não foram respondidos, 12 profissionais se negaram a participar da pesquisa, três desistiram e 15 aceitaram participar da amostra de juízes no primeiro painel de validação da pesquisa. O segundo 
painel ocorreu entre maio e junho de 2016 mediante envio do link aos 15 participantes da primeira etapa, em que 13 docentes responderam o questionário até o final das fases de validação.

A avaliação dos indicadores empíricos pelos especialistas ocorreu por meio de uma plataforma construída nos formulários do google Docs, via internet. Assim, cada participante recebeu um convite com um link para acessar a plataforma. Ao clicar no link o especialista automaticamente era direcionado à plataforma e ao concordar em participar da pesquisa clicava no link da aquiescência do Termo de Consentimento Livre e Esclarecido (TCLE), contido na primeira página. Após a concordância o participante tinha acesso ao conteúdo do instrumento para avaliação de cada item. O instrumento foi submetido à validação de forma e conteúdo pelos especialistas por meio da Técnica Delphi. O grau de relevância de cada item do instrumento foi avaliado considerando os conceitos de clareza e pertinência/representatividade.

A concordância foi avaliada a partir da frequência com que as respostas repetiam em cada unidade. Avaliou-se a confiabilidade a partir da homogeneidade das respostas, estabilidade e equivalência. O Coeficiente de Validade de Conteúdo (CVC) foi utilizado para avaliar a Pertinência/Representatividade de cada item do instrumento como um todo. Os juízes utilizaram uma escala de 01 a 04 pontos para avaliar o nível de adequação da Pertinência/Representatividade, sendo $1=$ irrelevante, $2=$ pouco relevante, $3=$ relevante e $4=$ muito relevante. Os itens deveriam apresentar $\mathrm{CVC} \geq 0,80 .{ }^{17-18}$

O CVC foi calculado por meio das seguintes equações:

1) $\mathrm{Mx}=\Sigma \mathrm{x} / \mathrm{J}$, onde $\mathrm{Mx}$ representa a média dos valores de cada item, $\Sigma x$ corresponde ao somatório dos valores obtidos na escala Likerte J é o número de juízes;

2) $\mathrm{CVCi}=\mathrm{Mx} / \mathrm{Vmáx}$, onde CVCi é o CVC de cada item e Vmáx é o valor máximo que cada item pode ter na escala Likert (quatro pontos);

3) CVCt = Mcvci, onde CVCt é o CVC total do questionário e Mcvci representa a média dos coeficientes de validade de conteúdo dos itens do questionário. 
O erro devido a possíveis vieses dos juízes foi de $2,284 \times 10-{ }^{18}$ e não foi considerado no cálculo do CVC, uma vez que foi próximo de zero. Para avaliar a Clareza da Linguagem foi utilizada a porcentagem de concordância. Os juízes utilizaram uma escala de 01 a 02 pontos para avaliar o nível de adequação da Clareza da Linguagem dos itens, sendo 1 =Inadequado e 2 = Adequado. A concordância para cada item foi calculada com o número de juízes que consideraram o item adequado dividido pelo número total de juízes, multiplicado por $100 .{ }^{18}$

O projeto foi encaminhado para análise e parecer do Comitê de Ética em pesquisa da Universidade Federal de Juiz de Fora e após aprovação por meio do parecer 1.324.662, em 16 de novembro de 2015, foi iniciada a etapa de validação do instrumento com os professores que aceitaram participar como juízes, considerando as sugestões fornecidas como confidenciais e respeitando a Resolução 466/2012 do Conselho Nacional de Saúde do Ministério da Saúde, que trata de pesquisas realizadas com seres humanos.

\section{Resultados}

O instrumento foi elaborado por meio de uma pesquisa nas fichas clínicas recomendadas pelo Ministério da Saúde e do Centro Latino-Americano de Perinatologia Saúde da Mulher e Reprodutiva da Organização Pan Americana da Saúde/Organização Mundial da Saúde. Esta decisão considerou a necessidade de adaptar um material abrangente, de forma que o profissional tenha segurança para acompanhar com qualidade o pré-natal de risco habitual na Atenção Básica. Dentre os documentos do Ministério da Saúde foram consultadas as fichas de pré-natal no Manual de Pré-Natal e Nascimento, Cadernetas da Gestante de 2014 e 2015, Protocolo de Atenção à Saúde da Mulher de 2015 e 2016, Caderno de Humanização do Parto e do Nascimento de 2014.

A construção do instrumento seguiu as etapas apresentadas pela Figura 2.

Figura 2 - Construção do Instrumento 
Seleção das fichas clínicas de pré-natal do Ministério da Saúde e do Centro Latino Americano de Perinatologia seguida de levantamento bibliográfico dos itens que devem constar na ficha clínica de pré-natal; Adaptação de um novo instrumento a partir da escolha criteriosa de itens.

Contratação de um especialista em designer gráfico para confeccionar a ficha no programa Corel Draw, após criação de esboço no editor de texto Word.

Construção de uma plataforma no Google Docs para disponibilizar a ficha com os itens a serem submetidos à validação através do envio de convites com links contendo o instrumento para os especialistas realizarem a avaliação.

Ressalta-se que a elaboração final da ficha clínica validada no primeiro painel ocorreu após a criação de um esboço no Word, que foi entregue para um designer confeccionar o modelo, sendo essa etapa realizada duas vezes a fim de aperfeiçoar a distribuição dos espaços entre os itens e acrescentar informações sugeridas pelos especialistas.

A primeira rodada da validação ocorreu em fevereiro de 2016 por meio da técnica Delphi, sendo constituída por 15 enfermeiros docentes de universidades públicas do Brasil, especialistas em Enfermagem Obstétrica, em que 10 apresentavam título de Doutor e 5 Pós-Doutorado. A experiência na área de ensino mínima foi de 9 anos, máxima de 38 anos e a média de 22 anos; a atuação mínima na área de Saúde da Mulher foi de 14 anos, máxima de 38 anos e a média de 24 anos; a atuação no pré-natal teve um período mínimo de 5 anos, máximo de 38 anos e a média de 20 anos.

Dentre os obstáculos pode-se citar a dificuldade de selecionar a amostra, posto que foi necessário fazer uma vasta busca em currículos da Plataforma Lattes e posteriormente procurar os e-mails. Quanto aos benefícios, destaca-se a oportunidade de realizar um estudo com profissionais de todas as regiões do Brasil com liberdade para emitirem opiniões e sugestões viáveis ao aprimoramento do trabalho mesmo estando distantes das pesquisadoras. Salienta-se que a amostra final de peritos alcançou todas as regiões do país: Norte (Acre): 1; Centro Oeste 
(Brasília, Mato Grosso do Sul): 3; Nordeste (Maranhão): 1; Sudeste (São Paulo, Rio de Janeiro): 6; Sul (Rio Grande do Sul): 4.

O segundo painel foi realizado em junho de 2016, com a participação de 13 especialistas que responderam a rodada anterior, sendo constituído pelos itens que não alcançaram a validação no painel 1 ou que foram sugeridos ajustes. Em relação à clareza e compreensão, foi observado Coeficiente de Validação de Conteúdo Total $=0,97$. Todos os itens apresentaram Coeficiente de Validação de Conteúdo acima do ponto de corte estabelecido, embora alguns peritos tenham sinalizado a necessidade de ajustes. O valor mínimo observado foi de 0,85 . Em relação à relevância, foi observado Coeficiente de Validação de Conteúdo Total = 0,84. O valor mínimo observado foi de 0,73 .

\section{Discussão}

A confecção da versão final do trabalho considerou o rigor e a acurácia das sugestões disponibilizadas no consolidado dos resultados quanto a face e layout para oferecer aos profissionais um material organizado, de fácil preenchimento e com boa visualização dos blocos. Sabe-se que em pesquisas de validação com mais de cinco peritos o coeficiente de validação encontrado deve ser igual ou superior a $80 \%$, o que confirma o êxito dessa pesquisa. ${ }^{19-20}$

Além disso, o emprego de uma ficha clínica construída a partir de um trabalho de validação, viabiliza uma assistência segura ao paciente com ênfase em registros completos em conformidade com o código de ética profissional e em consonância com o protocolo de segurança do paciente. Além disso, o prontuário configura-se como um documento passível de ser submetido a auditorias, pode ser solicitado pela justiça ou pelo paciente por se tratar de um documento, que reflete a qualidade da assistência e a segurança do paciente. ${ }^{21}$

O aperfeiçoamento da ficha englobou o acompanhamento do pré-natal do (a) parceiro (a) escolhido pela gestante, independentemente de ser ele o genitor, uma vez que respeitou a 
diversidade de gêneros e as novas conformações de famílias. Assim, o (a) companheiro(a) selecionado pela gestante ao ser inserido nas consultas de pré-natal poderá ampliar os cuidados com a sua saúde e compreender as mudanças que a parceira vivenciará na gravidez, aumentando a cumplicidade entre o casal. ${ }^{22}$

Dessa forma, todas as adaptações realizadas no instrumento até a confecção da versão final da ficha clínica foram ancoradas em evidências científicas e em um designer prático para otimizar o processo de trabalho do profissional que atua na Atenção Básica dividido em: Identificação da gestante; Antecedentes pessoais; Antecedentes familiares; Antecedentes obstétricos; Antecedentes ginecológicos; História de aleitamento; História nutricional; Atividade física; Gestação atual; Saúde bucal; Mamas; Condições obstétricas; Exames; Consulta de pré-natal; Quadro destinado às consultas de pré-natal; Gráficos de acompanhamento gestacional; Pré-natal do parceiro(a); Exames do parceiro(a); Parto/Aborto; Recém-nascido; Alta materna do local de parto; Alta recém-nascido.

A representação gráfica da ficha clínica ocorreu em folha A4 ocupando cinco (5) páginas, porém para a prática clínica recomenda-se a disposição em folha A3 para reduzir o volume de papéis no prontuário. O modelo final poderá ser consultado na dissertação intitulada "Validação de uma ficha clínica para a consulta de pré-natal às gestantes de risco habitual: Uma proposta de enfermeiras” por meio do endereço http://www.ufjf.br/pgenfermagem/files/2010/05/Disserta\%C3\%A7\%C3\%A3o-Daniela-AparecidaAlmeida-Duque.pdf.

O desenvolvimento de materiais para qualificar o cuidado em saúde consiste em uma estratégia para destacar a realização de consultas pelo Enfermeiro na Atenção Básica e corrobora com a nova Resolução do COFEN 606 de 2019, a qual regulamenta a atuação da enfermagem em empreendimentos na forma de consultórios e clínicas privadas. Salienta-se que a assistência em consultórios e clínicas deve apoiar-se na sistematização da assistência em 
13 I Duque, DAA, Fernandes, BM

enfermagem e ser comprometida com os registros em prontuários convencionais ou eletrônicos. ${ }^{23}$

Conclui-se que este trabalho atendeu todas as fases propostas pelo método, tal como ocorreu em outros estudos que auxiliaram na compreensão das etapas durante o percurso de validação. Além disso, encontra-se com layout e conteúdo em um formato organizado para direcionar e padronizar as consultas de pré-natal.

\section{Considerações finais}

Ao final dessa investigação evidenciou-se que a ficha clínica validada é uma tecnologia leve-dura passível de ser utilizada por Enfermeiras (os) e por outras categorias profissionais que realizam consultas de pré-natal na Atenção Primária à Saúde, constituindo-se como um instrumento para a consulta de pré-natal às gestantes de risco habitual na perspectiva de uma assistência de qualidade, capaz de contribuir com a redução da morbimortalidade materna e fetal no Brasil.

No decorrer da realização da validação da ficha constatou-se o envolvimento, a satisfação e o compromisso das especialistas ao colaborarem com a pesquisa e as expectativas em relação à contribuição do resultado final para as (os) profissionais que atuam no Sistema Único de Saúde. Ademais foi evidenciado a importância da ficha clínica como uma tecnologia na formação dos acadêmicos de enfermagem que poderão utilizá-la nos atendimentos de pré-natal em aulas práticas.

A limitação da pesquisa foi a insuficiência de tempo para aplicar a ficha clínica a uma amostra de profissionais, de modo a mensurar as dimensões do trabalho no cotidiano da Atenção Primária à Saúde.

\section{Referências}

1. Ministério da Saúde (BR). Atenção ao pré-natal de baixo risco. Série A. Normas e manuais técnicos. 
Cadernos de atenção básica, nº 32 [Internet]. Brasília (DF): Ministério da Saúde; 2012 [acesso em 2020 abr 15]. Disponível em: http://bvsms.saude.gov.br/bvs/publicacoes/cadernos_atencao_basica_32_prenatal.pdf

2. Alkema L, Chou D, Hogan D, Zhang S, Moller AB, Gemmill A, et al. Global, regional, and national levels and trends in maternal mortality between 1990 and 2015, with scenario-based projections to 2030: a systematic analysis by the UN Maternal Mortality Estimation Inter-Agency Group. The Lancet [Internet]. 2016 [cited 2020 mar 02];387(10017):462-74. Available from: https://reader.elsevier.com/reader/sd/pii/S0140673615008387?token=408BEC3127E6FEDE1C30F4618014B 3CACBF47254B61B88810CBBB491273019A7D082732C1AA0259E8D772A8F617D8B3E

3. Corrêa VAF, Acioli S, Tinoco TF. Cuidado do enfermeiro na Estratégia Saúde da Família: práticas e fundamentações teóricas. Rev Bras Enferm. 2018;71(6):2939-39. doi: https://doi.org/10.1590/0034-71672018-0383

4. Varela AR, Schneider BC, Bubach S, Silveira MF, Bertoldi AD, Duarte LSM, et al. Mortalidade fetal, neonatal e pós-neonatal e fatores associados na coorte de nascimentos de 2015 de Pelotas, Rio Grande do Sul, Brasil. Cad Saúde Pública. 2019;35(7):e00072918. doi: https://doi.org/10.1590/0102-311x00072918

5. Cunha AC, Lacerda JTD, Alcauza MTR, Natal S. Avaliação da atenção ao pré-natal na Atenção Básica no Brasil. Rev Bras Saúde Mater Infant. 2019;19(2):447-58. doi: http://dx.doi.org/10.1590/180693042019000200011

6. Alves MCOM, Rodrigues EOMA. Análise da adesão ao atendimento gravídico puerperal das mulheres de uma unidade básica de saúde. Rev Recien (Online). 2017;7(20):91-104. doi: https://doi.org/10.24276/rrecien2358-3088.2017.7.20.91-104

7. Andrade UV, Santos JB, Duarte C. A percepção da gestante sobre a qualidade do atendimento prénatal em UBS, Campo Grande, MS. Rev Psicol Saúde [Internet]. 2019 [acesso em 2020 abr 15];11(1):53-61. Disponível em: http://pepsic.bvsalud.org/scielo.php?script=sci_arttext\&pid=S2177-093X2019000100004

8. Nunes FBBF, Carneiro ICC, Prudêncio OS, Mamede FV. Evolução de indicadores maternos a partir do sistema de informação sobre nascidos vivos. Rev Enferm UFPE On Line [Internet]. 2016 [cited 2020 Mar 02];10(2 Suppl):771-9. Available from: https://periodicos.ufpe.br/revistas/revistaenfermagem/article/view/11019

9. CONSELHO FEDERAL DE ENFERMAGEM. Resolução COFEN no 159/1993, revogada pela Resolução Cofen no 544/2017. Dispõe sobre a consulta de Enfermagem. Rio de Janeiro: COFEN, 1983. Disponível em: http://www.cofen.gov.br/resoluo-cofen-1591993_4241.html. Acesso em: 02 mar. 2020.

10. Silva AA, Jardim MJA, Rios CTF, Fonseca LMB, Coimbra LC. Pré-natal da gestante de risco habitual: potencialidades e fragilidades. Rev Enferm UFSM [Internet]. 2019 [cited 2020 mar 02];9(15):1-20. Available from: https://periodicos.ufsm.br/reufsm/article/view/32336

11. Ministério da Saúde (BR), Secretaria de Ciência, Tecnologia e Insumos Estratégicos, Departamento 
de Ciência e Tecnologia. APPMS: Agenda de Prioridades de Pesquisa do Ministério da Saúde [Internet]. Brasília (DF): Ministério da Saúde; 2018 [acesso em 2020 abr 15];26 p. Disponível em: http://bvsms.saude.gov.br/bvs/publicacoes/agenda_prioridades_pesquisa_ms.pdf

12. Guimarães WSG, Parente RCP, Guimarães TLF, Garnelo L. Acesso e qualidade da atenção pré-natal na Estratégia Saúde da Família: infraestrutura, cuidado e gestão. Cad Saúde Pública. 2018;34(5):1-13. doi: https://doi.org/10.1590/0102-311x00110417

13. Sabino LMM, Brasil DRM, Caetano JA, Santos MCL, Alves MDS. Uso de tecnologia leve-dura nas práticas de enfermagem: análise de conceito. Aquichan. 2016;16(2):230-9. doi: http://dx.doi.org/10.5294/aqui.2016.16.2.10

14. Polit DF, Beck CT. Fundamentos de pesquisa em enfermagem: avaliação de evidências para a prática da Enfermagem. $7^{\text {a }}$ ed. Porto Alegre: Artmed; 2011.

15. Revorêdo LS, Maia RS, Torres GV, Maia EMC. O uso da técnica Delphi em saúde: uma revisão integrativa de estudos brasileiros. Arq Ciênc Saúde. 2015;22(2):16-21. doi: https://doi.org/10.17696/23183691.22.2.2015.136

16. Baldin N, Munhoz BEM. Snowball (bola de neve): uma técnica metodológica para pesquisa em educação ambiental comunitária. In: X Congresso de Educação - EDUCERE. I Seminário Internacional de Representações Sociais, Subjetividade e Educação - SIRSSE [Internet]. 2011 nov 07-10; Curitiba. Curitiba: Pontifícia Universidade Católica do Paraná; 2011 [acesso em 2020 mar 02]. p. 329-41. Disponível em: https://docplayer.com.br/1714932-Snowball-bola-de-neve-uma-tecnica-metodologica-para-pesquisaem-educacao-ambiental-comunitaria.html

17. Fidalgo ÁM, Scalonb JD. Uso dos métodos Mantel-Haenszel para a detecção do funcionamento diferencial dos itens e software relacionado. Psicol Reflex Crit. 2012;25(1): 60-8. doi: http://dx.doi.org/10.1590/S0102-79722012000100008

18. Lobiondo-Wood G, Haber J. Pesquisa em enfermagem: métodos, avaliação crítica e utilização. $4^{\mathbf{a}}$ ed. Rio de Janeiro: Guanabara-Koogan; 2001. Desenhos não experimentais; p. 110-21.

19. Pinto RH, Senna SM, Vasconcelos EMR, Leal LP, Santos CR, Sousa SM. Construção e validação do instrumento para avaliar o estresse em professores. Rev Enferm UFPE On Line. 2018;12(9):2283-92. doi: https://doi.org/10.5205/1981-8963-v12i9a235121p2283-2292-2018

20. Dupim SLM, Ribeiro LCC, Alves WM. Construção e validação de um instrumento sobre gestão do Sistema Único de Saúde. Rev Gest Saúde (Brasília) [Internet]. 2016 [acesso em 2020 mar 02];3(3):1191-99. Disponível em: https://dialnet.unirioja.es/servlet/articulo?codigo=5658764

21. Morais CGX, Batista EMS, Castro JFL, Assunção SS, Castro GMO. Registros de enfermagem em prontuário e suas implicações na qualidade assistencial segundo os padrões de acreditação hospitalar: um novo olhar da auditoria. Rev Acreditação. 2015 [acesso em 2020 mar 02];5(9):64-84. Disponível em: 
https://dialnet.unirioja.es/servlet/articulo?codigo $=5626617$

22- Queiroz ENS, Santos AA, Melo KKO, Magalhães AYF, Martins LLF, Portela FBS. Avaliação da adesão ao pré-natal do parceiro: impacto no trinômio. Braz J Health Rev [Internet]. 2019 [acesso em 2020 mar 02];2(5):4835-41. doi: https://doi.org/10.34119/bjhrv2n5-080

23- CONSELHO FEDERAL DE ENFERMAGEM. Resolução COFEN nำ 606, de 5 de abril de 2019. Inclui na Resolução Cofen $\mathrm{n}^{\circ}$ 568, de 9 de fevereiro de 2018, anexos contendo modelo de Requerimento de Cadastro de Consultório e de Clínicas de Enfermagem e modelo de Registro de Consultório e de Clínicas de Enfermagem, no âmbito dos Conselhos Regionais de Enfermagem. Brasília (DF): COFEN, 2019. Disponível em: http://www.cofen.gov.br/resolucao-cofen-no-606-2019_70088.html. Acesso em: 02 mar. 2020.

\section{Autor correspondente}

Daniela Aparecida Almeida Duque

E-mail: danielaalmeidaduque@gmail.com

Endereço: Rua Carlos Moreira, Lima Duarte, MG

CEP: $36140-000$

\section{Contribuições de Autoria}

\section{1 - Daniela Aparecida Almeida Duque}

Responsável pela pesquisa e construção da dissertação

\section{2 - Betânia Maria Fernandes}

Professora responsável por orientar está pesquisa no Programa de Pós-Graduação Stricto Sensu do Mestrado Acadêmico em Enfermagem da Universidade Federal de Juiz de Fora

\section{Como citar este artigo}

Duque, DAA, Fernandes, BM. Construção e validação de ficha clínica para acompanhamento de prénatal de risco habitual. Rev. Enferm. UFSM. 2020 [Acesso em: Ano Mês Dia]; vol.10 e48 1-16. DOI:https://doi.org/10.5902/2179769239763 